\title{
The $\kappa-\mu$ Extreme Distribution: Theory and Applications
}

\author{
Guilherme Silveira Rabelo, Ugo Silva Dias and Michel Daoud Yacoub
}

\begin{abstract}
Resumo-Este artigo apresenta a distribuição de desvanecimento $\kappa-\mu$ Extrema, utilizada para descrever a propagação de rádio móvel sob condições severas de desvanecimento. Tais condições ocorrem principalmente em ambientes confinados, embora podem ser encontradas em áreas abertas cujos dispersores movem-se rapidamente. São realizados ajustes para dados de campo presentes na literatura e outros coletados a partir de medições realizadas pelos autores. Resultados de comparação entre a distribuição $\kappa-\mu$ Extrema e outro modelo proposto recentemente, chamado Two-Ray, mostram que a distribuição $\kappa-\mu$ Extrema é mais flexível e adequada para modelar condições severas de desvanecimento.
\end{abstract}

Palavras-Chave - Distribuição $\kappa$ - $\mu$ Extreme, Distribuição TwoRay, Desvanecimento Hyper-Rayleigh, Canais com desvanecimento, Medidas de campo.

Abstract-This paper presents the $\kappa-\mu$ Extreme fading distribution, which is used for characterizing mobile radio propagation under severe fading conditions. Such conditions occur specially in enclosed environments, although they may be found in open areas in which the scatters move rapidly. The distribution is adjusted to field data extracted from the literature and also to data collected from measurements performed by the authors. Comparison with another model recently proposed elsewhere, namely the Two-Ray model, shows that the $\kappa-\mu$ Extreme distribution is flexible and suitable to better model the severe fading conditions.

Keywords- $-\kappa-\mu$ Extreme Distribution, Two-Ray Distribution, Hyper-Rayleigh Fading, Fading Channels, Field trials.

\section{INTRODUCTION}

Wireless communication services expand at a rapid pace and their applications seem limitless. Previously restricted to outdoor environments, they soon reached indoor ones, including shopping malls, airports, and, more recently, enclosed environments, namely airplanes, trains, and buses. Along with those services, other wireless applications such as in Wireless Sensor Networks (WSN) [1] and Wireless Local Area Networks (WLAN) [2] have been also widely deployed throughout a variety of possible scenarios.

In this context, the characterization of the wireless propagation conditions, in particular the fading conditions, continue to raise interest [1], [2], [3]. Nevertheless, as opposed to those initial scenarios, namely outdoor and indoor, for which the propagation conditions are reasonably well known, those for enclosed environments have yet to be better understood [3], [4]. Questions arise that concern the appropriateness of the largely used small scale fading distributions, such as Nakagami$m$ [5], Weibull [6], and others, as applied to the enclosed

The authors are with the Wireless Technology Laboratory (WissTek), Department of Communications, School of Electrical and Computer Engineering, University of Campinas, PO Box 6101, 13083-852, Campinas, SP, Brazil. Email: [rabelo,ugo,michel]@wisstek.org environments. This question has been recently addressed in [7].

Enclosed environments are known to be characterized by very severe fading conditions. Unlike the homogeneous ones, whose different combinations of large number of multipath components lead to known fading channels, enclosed environments may present only a few number of dominant paths, therefore rendering the approximation by the Central Limit Theorem [3] inappropriate. As a consequence, the radio channel in such conditions are not correctly described by those known distributions [3], [7]. Additionally, traditional propagation models (e.g, Plane Earth [8]) predict that direct and reflected waves may be combined to yield nulls at some reception points. With only few waves being combined, the occurrence of nulls becomes frequent and must be accounted for. In [7], the severe fading conditions - worse than that predicted by the Rayleigh case - in enclosed environments was named hyper-Rayleigh fading [3], [7], [9].

An initial approach towards describing mobile propagation under the mentioned severe fading conditions was carried out in [3]. In that work, a new probability distribution, the TwoRay distribution, was proposed as a worst-case scenario fading model. The Two-Ray model consists of a special case of the Two-Ray With Diffuse Power (TWDP) model [4], assuming (i) no diffuse power and (ii) the two dominant components with identical magnitudes and random phase [3].

The Two-Ray model is indeed simple, but has some limitations: it neither predicts a received signal with magnitude larger than twice the magnitude of a single dominant component nor is flexible to adjust to conditions other than the limited tworay scenario. In real world, several degrees of severe fading or hyper-Rayleigh - conditions exist that will certainly depart from that predicted by the Two-Ray model.

This work assesses the feasibility of the use of the $\kappa-\mu$ Extreme distribution - a particular case of the $\kappa$ - $\mu$ distribution, obtained for extreme values of its parameters [10] - for describing mobile propagation under severe fading conditions, including the conditions one might experience within enclosed environments.

The remainder of this paper is structured as follows. In Section II, the $\kappa-\mu$ Extreme distribution is revisited, including its derivation from the $\kappa-\mu$ distribution. In Section III, plots of Rayleigh, Two Ray, and $\kappa-\mu$ Extreme CDFs are compared. In Section IV, a curve fitting comparison between the $\kappa$ $\mu$ Extreme model and the Two-Ray model is performed, using field measurements presented in [7]. In Section V, a validation through new field measurements is done in order to demonstrate the use of the $\kappa$ - $\mu$ Extreme model as a general 
severe fading model. Finally, in Section VI, conclusions are presented.

\section{The $\kappa-\mu$ EXTREME DistribUtion REVISITED}

The $\kappa-\mu$ Extreme distribution was first proposed in [10] and arises as a particular case of $\kappa-\mu$ distribution, for which the parameters assume extreme values, as shall be revisited next. The $\kappa-\mu$ distribution is a general fading distribution that represents the small-scale variations of the fading signal under line-of-sight (LOS) and multiple clusters of multipath conditions [10]. As its name implies, it is written in terms of two physical parameters, namely $\kappa$ and $\mu$. The parameter $\kappa>0$ concerns the ratio between the total power of the dominant components and the total power of the scattered waves, whereas the parameter $\mu>0$ is related to the multipath clustering. It includes as special cases important other distributions such as Rice $(\mu=1)$ and Nakagami- $m$ $(\kappa \rightarrow 0)$. Its flexibility renders it suitable to better fit field measurement data in a variety of scenarios, both for low[10] and high-order statistics [11]. For a fading signal with envelope $R$, with $\hat{r}=\sqrt{E\left(R^{2}\right)}$ being the rms value of $R$, the $\kappa-\mu$ envelope probability density function (PDF) $f_{\mathrm{P}}(\rho)$ of the normalized envelope $\mathrm{P}=R / \hat{r}$ is given by [10]

$$
\begin{aligned}
& f_{\mathrm{P}}(\rho)=\frac{2 \mu(1+\kappa)^{\frac{\mu+1}{2}}}{\kappa^{\frac{\mu-1}{2}} \exp (\mu \kappa)} \rho^{\mu} \exp \left[-\mu(1+\kappa) \rho^{2}\right] \\
& \times I_{\mu-1}[2 \mu \sqrt{\kappa(1+\kappa)} \rho],
\end{aligned}
$$

in which $\mu=E^{2}\left(R^{2}\right)(1+2 \kappa) / \operatorname{Var}\left(R^{2}\right)(1+\kappa)^{2}, I_{\nu}(\cdot)$ is the modified Bessel function of the first kind and order $\nu[12$, Equation 8.406] and $E(\cdot)$ and $\operatorname{Var}(\cdot)$ denote the expectation and variance operators, respectively. As detailed in [10], for a given Nakagami $m$ parameter, an infinite number of curves of the $\kappa-\mu$ distribution can be obtained for appropriate values of $\kappa$ and $\mu$. The mentioned parameter $m$, as well known, constitutes the inverse of the variance of the normalized power of the fading signal, i.e., $m=\operatorname{Var}^{-1}\left(\mathrm{P}^{2}\right)$, and is given in terms of $\kappa$ and $\mu$ as [10]

$$
m=\frac{\mu(1+\kappa)^{2}}{1+2 \kappa} .
$$

The $\kappa-\mu$ Extreme distribution is obtained by keeping $m$ constant and allowing $\kappa \rightarrow \infty$ (very strong LOS) and $\mu \rightarrow 0$ (very few multipaths). As a result, its normalized envelope $\operatorname{PDF} f_{\mathrm{P}}(\rho)$ is expressed as [10, Equation 16]

$f_{\mathrm{P}}(\rho)=\frac{4 m I_{1}(4 m \rho)}{\exp \left[2 m\left(1+\rho^{2}\right)\right]}+\left[1-\frac{\sqrt{2 m \pi}}{\exp (m)} I_{0.5}(m)\right] \delta(\rho)$,

where $\delta(\cdot)$ is the Dirac delta function. Using $I_{1 / 2}=$ $[\exp (m)-\exp (-m)] / \sqrt{2 \pi m}$ [12, Equation 8.447] in (3), the $\kappa-\mu$ Extreme normalized envelope PDF can be expressed in a simpler manner as

$$
f_{\mathrm{P}}(\rho)=\frac{4 m I_{1}(4 m \rho)}{\exp \left[2 m\left(1+\rho^{2}\right)\right]}+\exp (-2 m) \delta(\rho) .
$$

Its cumulative distribution function $(\mathrm{CDF})$ is obtained as

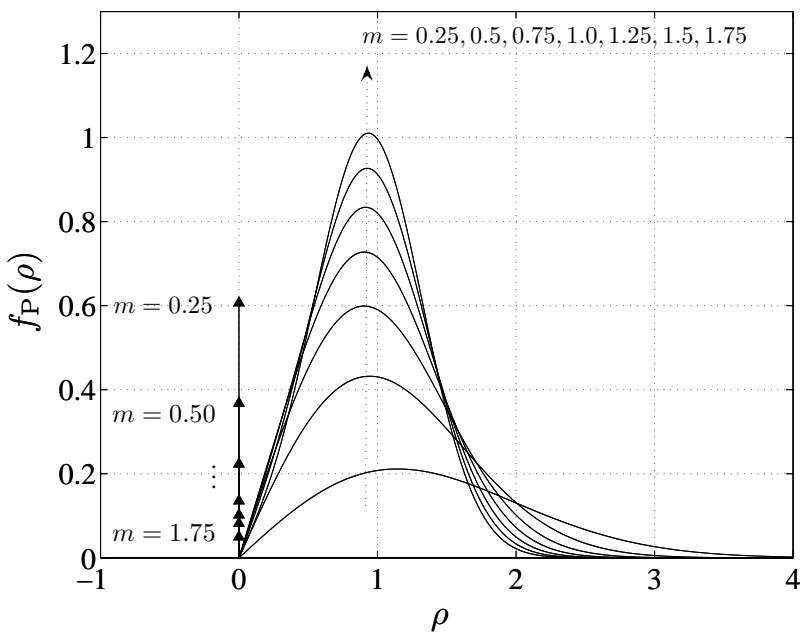

Fig. 1. $\quad \kappa-\mu$ Extreme probability density function.

$$
F_{\mathrm{P}}(\rho)=1-Q_{0}(2 \sqrt{m}, 2 \sqrt{m} \rho)
$$

in which

$$
Q_{0}(a, b)=a \int_{b}^{\infty} \exp \left(-\frac{x^{2}+a^{2}}{2}\right) I_{1}(a x) d x
$$

is the zeroth Marcum $Q$-function [13]. In Appendix I, a series representation for the $\kappa-\mu$ Extreme CDF is derived as

$$
F_{\mathrm{P}}(\rho)=1-\exp (-2 m) \sum_{k=0}^{\infty} \frac{(2 m)^{k+1}}{(k+1) ! k !} \Gamma\left(k+1,2 m \rho^{2}\right),
$$

where $\Gamma(\alpha, x)=\int_{x}^{\infty} e^{-t} t^{\alpha-1} d t$ is the incomplete Gamma Function [12, Equation 8.350.2]. Alternatively, by making use of $\Gamma(1+k, x)=k ! e^{-x} \sum_{n=0}^{k} \frac{x^{n}}{n !}$ [12, Equation 8.352.2] for integer values of $k$, the $\kappa-\mu$ Extreme CDF can be expressed by

$F_{\mathrm{P}}(\rho)=1-\exp \left[-2 m\left(1+\rho^{2}\right)\right] \sum_{k=0}^{\infty} \frac{(2 m)^{k+1}}{(k+1) !} \sum_{n=0}^{k} \frac{\left(2 m \rho^{2}\right)^{n}}{n !}$.

The $j$ th moment, $E\left(\mathrm{P}^{j}\right)$, of $\mathrm{P}$ is written in closed-form formula as

$$
E\left(\mathrm{P}^{j}\right)=\frac{j m \Gamma(j / 2)}{(2 m)^{\frac{j}{2}}} \times{ }_{1} F_{1}(1-j / 2 ; 2 ;-2 m),
$$

$j>0$ and $E\left(\mathrm{P}^{0}\right)=1$, in which ${ }_{1} F_{1}(\cdot ; \cdot ; \cdot)$ is the confluent hypergeometric function [14, Equation 13.1.2]. Of course, $E\left(\mathrm{R}^{j}\right)=\hat{r}^{j} E\left(\mathrm{P}^{j}\right)$. Interestingly, in the $\kappa-\mu$ Extreme distribution, the relation $m=\operatorname{Var}^{-1}\left(\mathrm{P}^{2}\right)$ is maintained. Figures 1 and 2 depict samples of the various shapes of the $\kappa-\mu$ Extreme PDF and CDF, $f_{\mathrm{P}}(\rho)$ and $F_{\mathrm{P}}(\rho)$, as functions of the normalized envelope, $\rho$, for different values of $m$. Note that the impulse at the origin indicates that a non nil probability exists for signal nulls. 


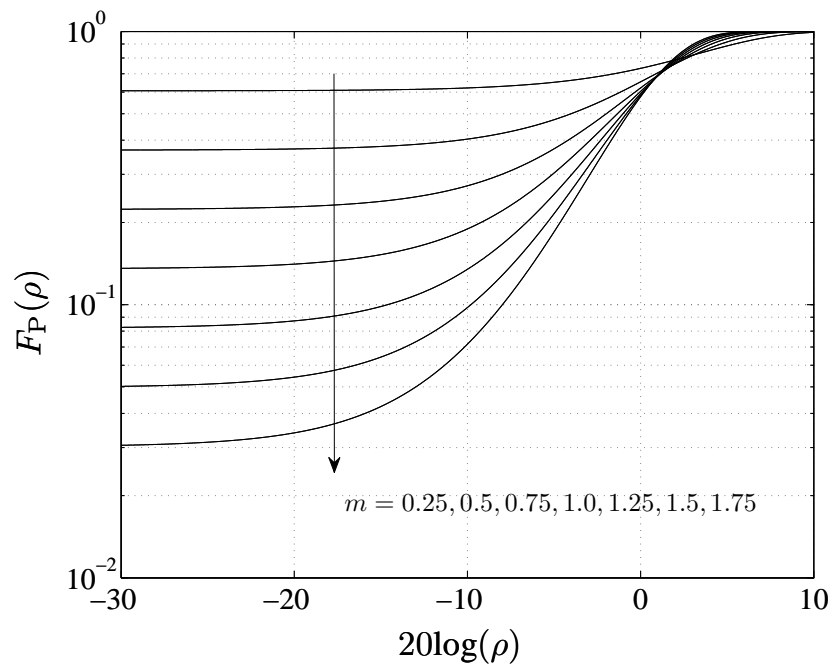

Fig. 2. $\kappa-\mu$ Extreme cumulative distribution function.

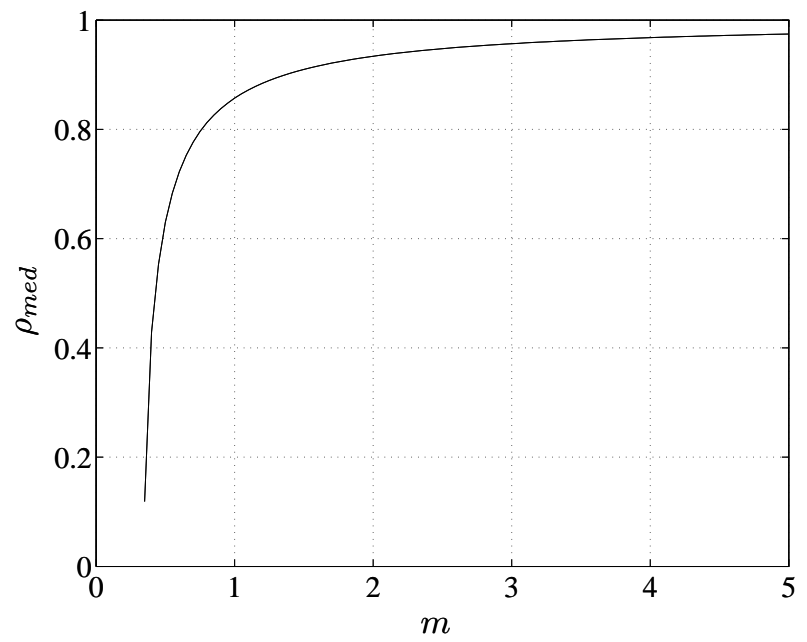

Fig. 3. $\kappa-\mu$ Extreme median as function of the parameter $m$.

\section{RAYleigh, Two-RAY, AND $\kappa-\mu$ ExtReme}

In [3], [7], the Two-Ray and Rayleigh curves were compared. Plotted as functions of the envelope normalized with respect to their median values, the Two-Ray CDF was shown to be above the Rayleigh CDF, justifying the name hyperRayleigh fading. It must noted, however, if the envelopes are normalized with respect to their rms values, these two curves would cross each other at a certain point. In this section, we compare the Rayleigh, Two-Ray, and $\kappa-\mu$ Extreme CDFs. In order to do so, first the median value of the $\kappa$ $\mu$ Extreme distribution must be determined. Unfortunately, no closed-form expression can be encountered. The equation $\rho_{\text {med }}=F_{\mathrm{P}}^{-1}(1 / 2)$ is found by numerical means and plotted as a function of $m$ in Figure 3. The plots of the three CDFs can be seen in Figure 4. Note that a number of hyper-Rayleigh situations can be found for the $\kappa-\mu$ Extreme case. In particular, for $m=1$, the following can be noted: below $0 \mathrm{~dB}$, the $\kappa-\mu$ Extreme curve lies above the Rayleigh curve, and above $0 \mathrm{~dB}$, it follows Rayleigh.

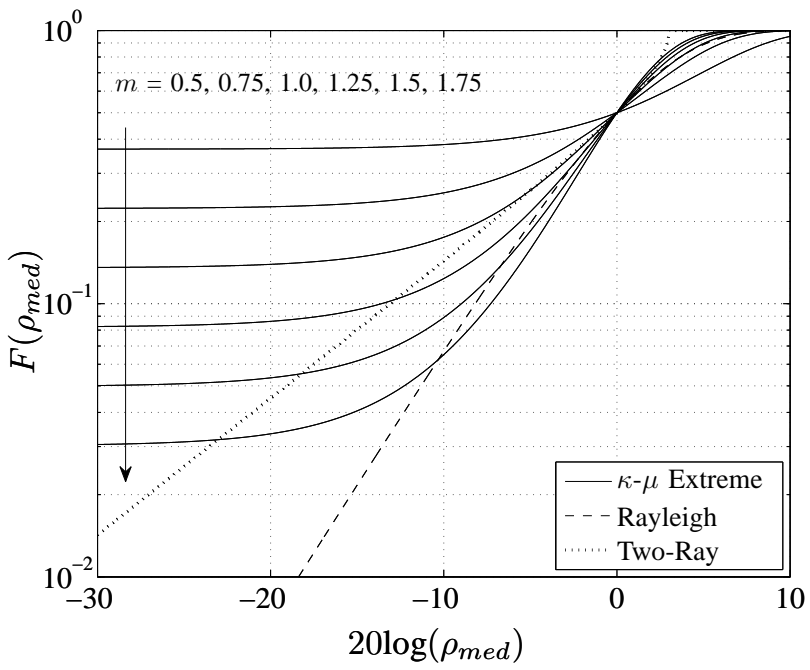

Fig. 4. Rayleigh, Two-Ray and $\kappa-\mu$ Extreme CDFs as function of the normalized envelope with respect to its median value.

\section{Application I: EnClosed EnVIRONMENTS}

In [7], field measurements were presented for wireless sensor applications in which the received signal experienced hyper-Rayleigh fadings. The fading data was collected aboard a large transport helicopter at the $2.4 \mathrm{GHz}$ ISM band and illustrated in Figure 4 of [7].

In order to compare the curve fitting performance of the $\kappa-\mu$ Extreme distribution and the Two-Ray distribution, the experimental points from these curves were carefully extracted and used to adjust the distributions. Figure 5 illustrates the collected data along with the Two-Ray distribution. In such a case, all curves are normalized with respect to its respective median $\left(\rho_{\text {med }}=r / r_{\text {med }}\right)$, therefore passing through coordinate $(0 \mathrm{~dB}$, $0.5)$.

Figures 6 to 10 show field measurement data from [7] for which the $\kappa-\mu$ Extreme CDF was adjusted to give its best fit. For comparison, the Two-Ray curves are also plotted. Table I presents the Mean Square Error (MSE) obtained from the fading data adjustment. The fitting was performed using logarithmic scale, in an attempt to better adjust the tail of the fading data. Despite the irregular behavior of fading data presented in Figures 6 to 10, it can been seen through Table I that, in three out of the five cases, the $\kappa-\mu$ Extreme distribution presented better agreement to the measured data than the TwoRay distribution.

For Fading data \#1 and \#2, Figures 6 and 7, respectively, the adjustment by the $\kappa-\mu$ Extreme distribution resulted in significant less MSE than the adjustment by the Two-Ray distribution. Specifically for Fading data \#1, the $\kappa-\mu$ Extreme distribution was able to well represent the tail of the measured data, as it can be seen in Figure 6. As for the Fading data $\# 2$, the tail of measured data presented extreme unexpected behavior, making impossible for both distributions to perform a suitable fit.

For Fading data \#3, illustrated in Figure 8, the $\kappa-\mu$ Extreme distribution also presented better adjustment performance than the Two-Ray distribution, although the MSE resulted from the 


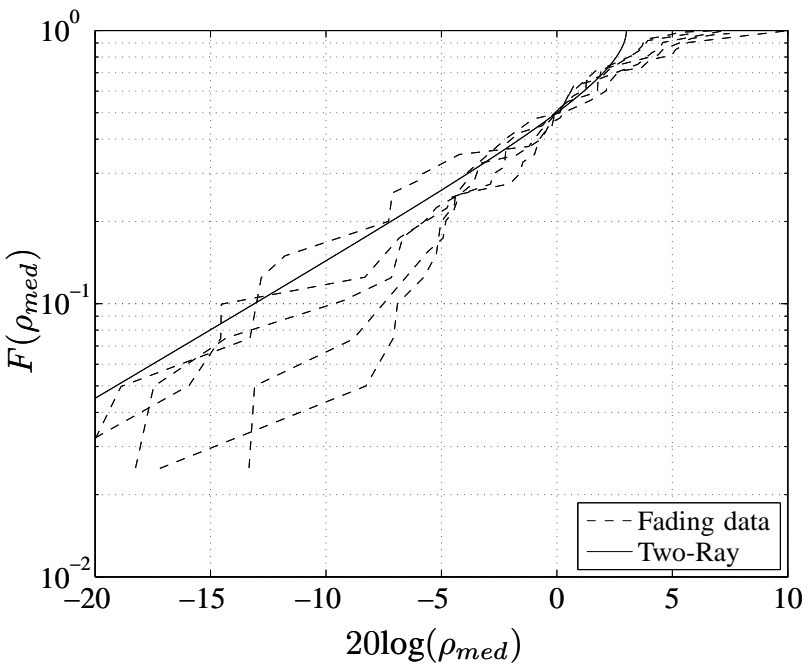

Fig. 5. Cumulative distribution of fading data presented in [7] and the TwoRay CDF.

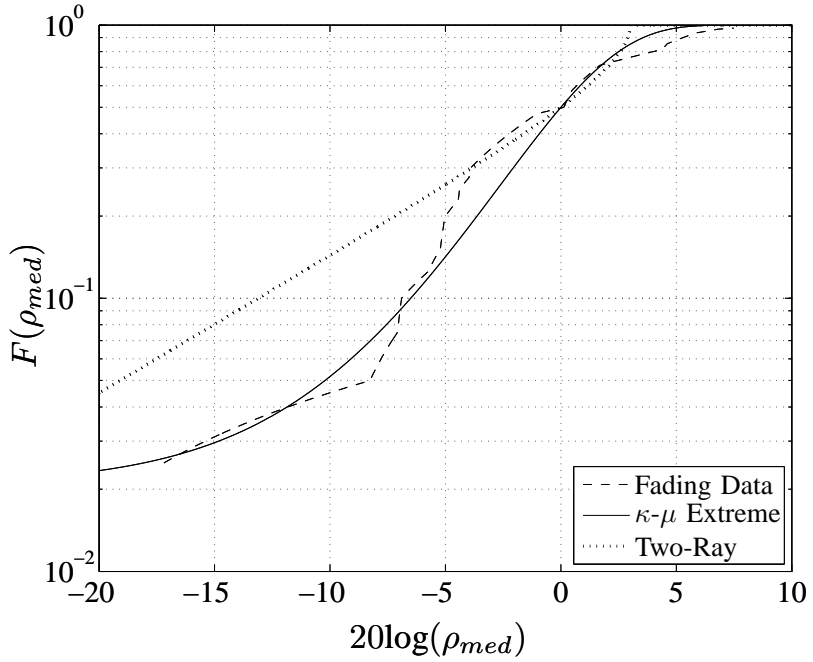

Fig. 6. The $\kappa-\mu$ Extreme distribution $(m=1.94)$ and the Two-Ray distribution adjusted to Fading data \#1.

adjustments did not differ in so great magnitude as in the previous cases. Similar to the Fading data \#2, the Fading data \#3 also presents an abrupt decay at the curve tail.

Finally, for Fading data \#4 and \#5, the Two-Ray presented better adjustment results than the $\kappa-\mu$ Extreme distribution. Despite the MSE results, both distributions presented similar agreement, as it can be seen through Figures 9 and 10 . Specifically for the values of $20 \log \left(\rho_{\text {median }}\right)>0$, which represent the cases of constructive composition of the received signal, the fading data's behavior was better represented by the $\kappa-\mu$ Extreme distribution, which occurred in all other cases.

The results pointed out two main limitations of the TwoRay model. The first one is the prediction of received signals whose envelope is restricted only to the interval $0 \leq r<2$. Consequently, for the cases of constructive composition of the received signal, which corresponds to the region where $20 \log \left(\rho_{\text {median }}\right)>0$, the Two-Ray distribution assumes such behavior which was not observed in any cases of fading data.

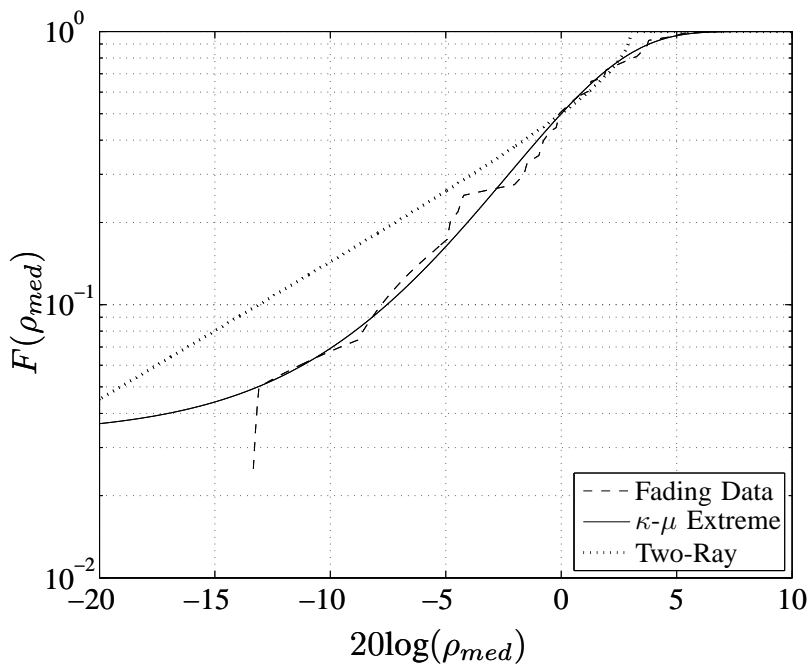

Fig. 7. The $\kappa-\mu$ Extreme distribution $(m=1.70)$ and the Two-Ray distribution adjusted to Fading data \#2.

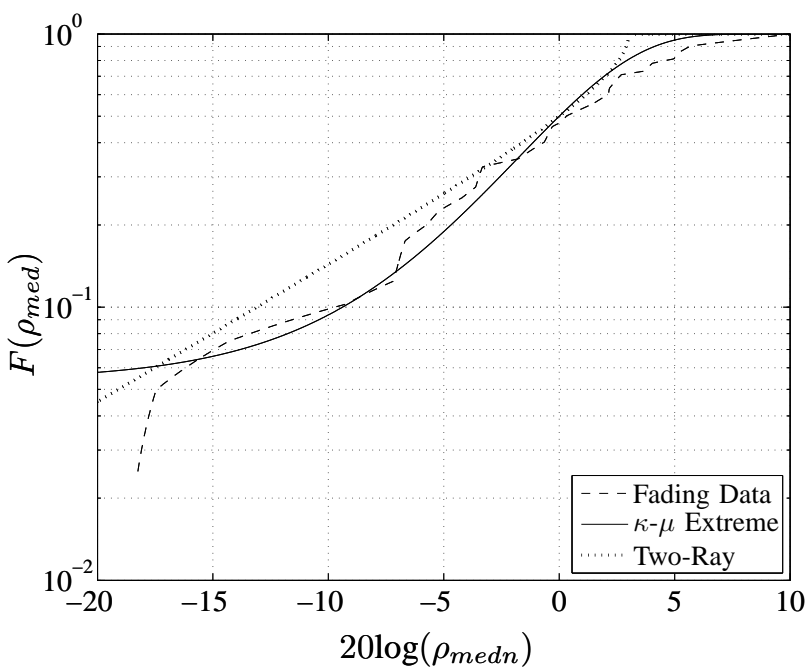

Fig. 8. The $\kappa-\mu$ Extreme distribution $(m=1.46)$ and the Two-Ray distribution adjusted to Fading data \#3.

The second main limitation is the lack of a fading parameter, which implies no flexibility for curve fitting. Therefore, the Two-Ray model shows itself suitable for representing only a few case of severe fading conditions. In the other hand, the fading parameter $m$ of the $\kappa-\mu$ Extreme distribution, whose calculation is straightforward given the fading data, allows greater flexibility to fit measured data in such cases.

TABLE I

Performance Comparison of Field Measurement Adjustment

\begin{tabular}{|c|c|c|c|}
\hline Curves & $m$ & $\kappa-\mu$ Extreme MSE & Two-Ray MSE \\
\hline Fading data \#1 & 1.94 & 0.0061 & 0.0946 \\
Fading data \#2 & 1.70 & 0.0014 & 0.0380 \\
Fading data \#3 & 1.46 & 0.0037 & 0.0099 \\
Fading data \#4 & 1.45 & 0.0102 & 0.0070 \\
Fading data \#5 & 1.33 & 0.0179 & 0.0045 \\
\hline
\end{tabular}




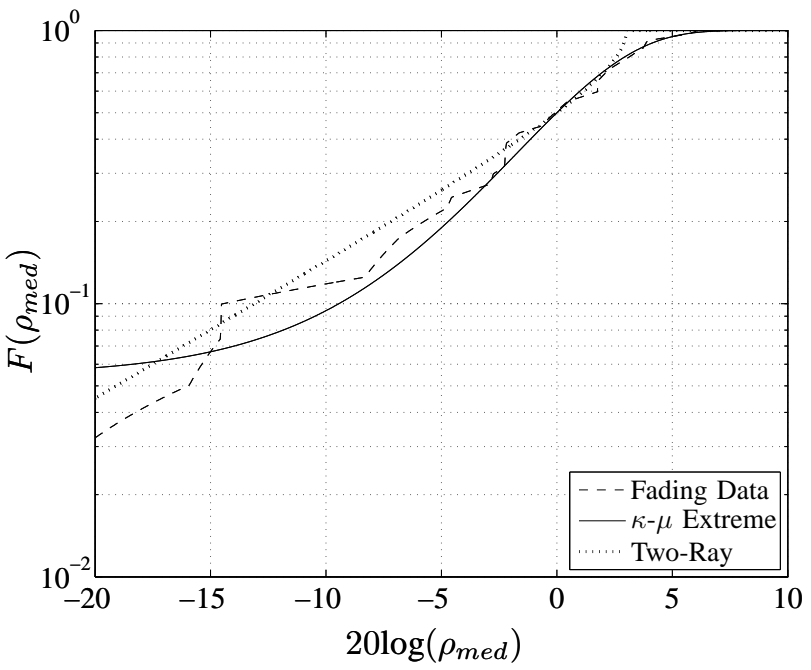

Fig. 9. The $\kappa-\mu$ Extreme distribution $(m=1.45)$ and the Two-Ray distribution adjusted to Fading data \#4.

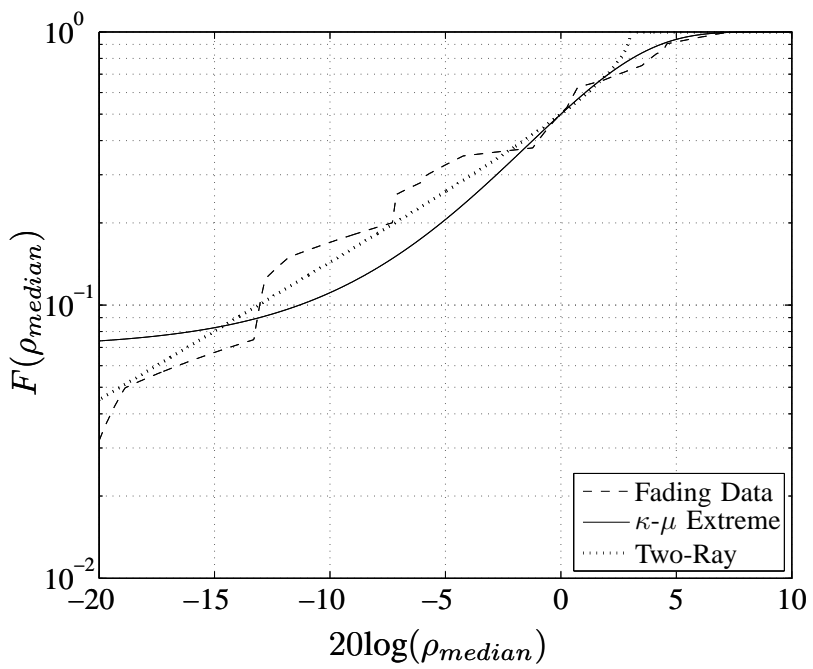

Fig. 10. The $\kappa-\mu$ Extreme distribution $(m=1.33)$ and the Two-Ray distribution adjusted to Fading data \#5.

\section{Application II: Other Severe Fading Conditions}

Several other situations for severe fading conditions may be found in practice. In order to investigate these conditions and validate the $\kappa-\mu$ Extreme model, a series of field trials were conducted at the University of Campinas (Unicamp), Brazil. To this end, a parking lot with cars aligned and moving vehicles was chosen. Both transmitter and receiver were placed below the height of the cars and a LOS condition was always in place. The mobile reception equipment was especially assembled for this purpose. Basically, the setup consisted of a vertically polarized omnidirectional receiving antenna, a low noise amplifier, a spectrum analyzer, data acquisition apparatus, a notebook computer, and a distance transducer for carrying out the signal sampling. The transmission consisted of a $\mathrm{CW}$ tone at $1.8 \mathrm{GHz}$. The spectrum analyzer was set to zero span and centered at the desired frequency, and its video output used as the input of the data-acquisition and processing

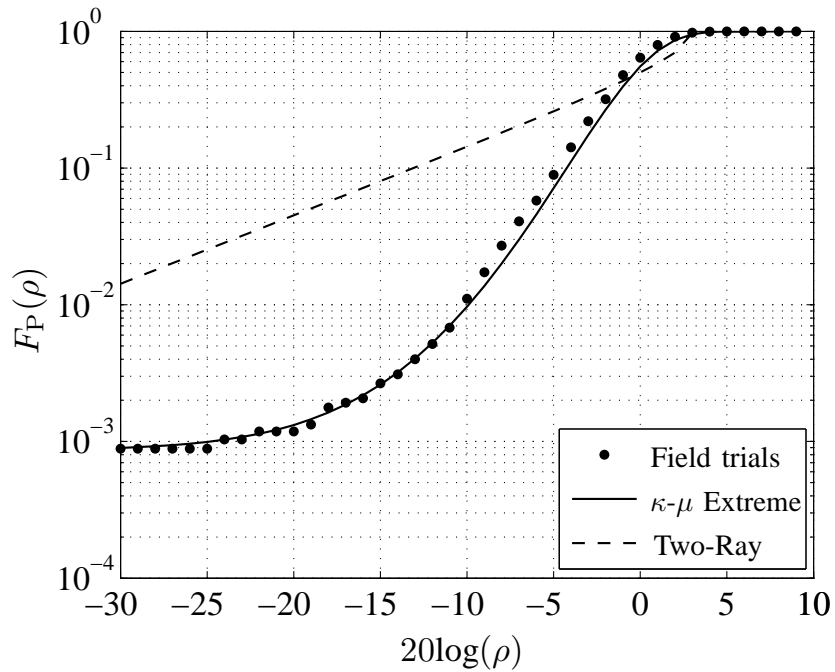

Fig. 11. Empirical versus theoretical cumulative distribution functions ( $m=$ $3.53)$.

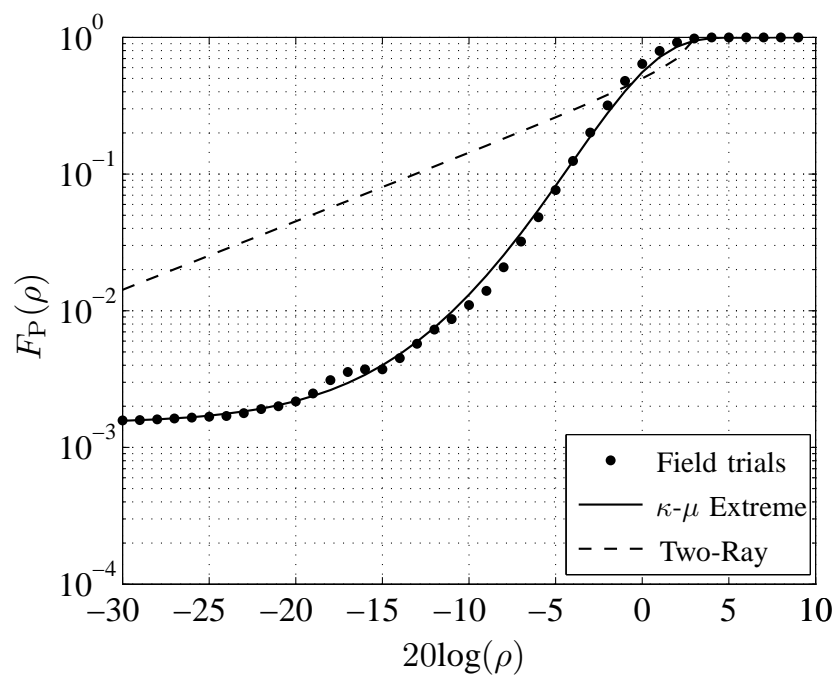

Fig. 12. Empirical versus theoretical cumulative distribution functions ( $m=$ $3.25)$.

equipment. The local mean was estimated by the moving average method, with the average being conveniently taken over samples symmetrically adjacent to every point. From the collected data, the fading parameter $m$ was estimated. In such a situation, the received signal was found to vary drastically. More specifically, due to the severe variation of the received signal, a great deal of points were found to be below the receiver sensitivity.

The empirical cumulative distribution function was compared against the corresponding theoretical formulas (8) and [3, Equation 5] and plotted as a function of the normalized envelope $\rho(\mathrm{dB})$ with the same parameter $m$ estimated from the experimental data. In addition, the MSE was computed for each case. Figures 11 and 12 show some sample plots comparing the experimental and theoretical CDF data. Table II presents the respective errors and empirical fading parameters. Observe the excellent fit and how the theoretical curves tend to keep track the tail of the empirical data. 
TABLE II

PERFORMANCE COMPARISON BETWEEN EMPIRICAL AND THEORETICAL DATA

\begin{tabular}{|c|c|c|c|}
\hline Field Trials & $m$ & $\kappa-\mu$ Extreme MSE & Two-Ray MSE \\
\hline Fig. 11 & 3.53 & 0.0030 & 1.1321 \\
Fig. 12 & 3.25 & 0.0017 & 0.8554 \\
\hline
\end{tabular}

\section{CONCLUSIONS}

This paper presented the $\kappa$ - $\mu$ Extreme fading distribution. Derived from the general $\kappa-\mu$ distribution for its parameters assuming extreme values, the $\kappa-\mu$ Extreme distribution presents a fading parameter which can be used for adjustment to experimental data. Adjustments to field measurements previously presented in literature have shown the flexibility of the $\kappa-\mu$ Extreme distribution to adequately fit to experimental data in severe fading conditions.

\section{APPENDIX I}

DERIVATION OF A SERIES EXPANSION FOR $\kappa$ - $\mu$ EXTREME CDF

By definition [15],

$$
\begin{aligned}
F_{\mathrm{P}}(\rho) & =\int_{-\infty}^{\rho} f_{\mathrm{P}}(\rho) d \xi \\
& =\int_{-\infty}^{\rho}\left(\frac{4 m I_{1}(4 m \xi)}{\exp \left[2 m\left(1+\xi^{2}\right)\right]}+\exp (-2 m) \delta(\xi)\right) d \xi
\end{aligned}
$$

Making use of $I_{1}(z)=\sum_{k=0}^{\infty} \frac{(z / 2)^{2 k+1}}{k !(k+1) !}$ [12, Equation 8.447], the $\kappa-\mu$ Extreme CDF becomes

$$
\begin{aligned}
& F_{\mathrm{P}}(\rho)= \\
& e^{-2 m}-4 m e^{-2 m} \int_{-\infty}^{\rho}\left(\sum_{k=0}^{\infty} \frac{(2 m \xi)^{2 k+1}}{k !(k+1) !} e^{-2 m \xi^{2}}\right) d \xi .
\end{aligned}
$$

As the term inside the integral is absolutely convergent,

$$
\begin{aligned}
& F_{\mathrm{P}}(\rho)= \\
& \quad e^{-2 m}-4 m e^{-2 m} \sum_{k=0}^{\infty} \int_{-\infty}^{\rho} \frac{(2 m \xi)^{2 k+1}}{k !(k+1) !} e^{-2 m \xi^{2}} d \xi .
\end{aligned}
$$

Solving the integral,

$$
\begin{aligned}
& F_{\mathrm{P}}(\rho)=e^{-2 m}-4 m e^{-2 m} \times \\
& \sum_{k=0}^{\infty} \frac{(2 m)^{2 k+1}}{k !(k+1) !}\left(2^{-2-k}\right) m^{-k-1}\left[k !-\Gamma\left(k+1,2 m \rho^{2}\right)\right]
\end{aligned}
$$

where $\Gamma(\alpha, x)=\int_{x}^{\infty} e^{-t} t^{\alpha-1} d t$ is the incomplete Gamma Function [12, Equation 8.350.2]. Rearranging Equation 13, the $\kappa-\mu$ Extreme CDF is finally obtained:

$$
F_{\mathrm{P}}(\rho)=1-e^{-2 m} \sum_{k=0}^{\infty} \frac{(2 m)^{k+1}}{(k+1) ! k !} \Gamma\left(k+1,2 m \rho^{2}\right) .
$$

\section{REFERENCES}

[1] J. Frolik and T. Weller, "Wireless sensor systems: an approach for a multiuniversity design course," Education, IEEE Transactions on, vol. 45, no. 2, pp. 135-141, 2002.

[2] N. Sarkar and E. Lo, "Indoor propagation measurements for performance evaluation of IEEE 802.11g," in Telecommunication Networks and Applications Conference, 2008. ATNAC 2008. Australasian, 2008, pp. $163-168$.

[3] J. Frolik, "A case for considering Hyper-Rayleigh fading channels," Wireless Communications, IEEE Transactions on, vol. 6, no. 4, pp. 1235-1239, 2007.

[4] G. Durgin, T. Rappaport, and D. de Wolf, "New analytical models and probability density functions for fading in wireless communications," Communications, IEEE Transactions on, vol. 50, no. 6, pp. 1005-1015, 2002.

[5] M. Nakagami, The m-Distribution - A General Formula of Intensity Distribution of Rapid Fading, W. C. Hoffman, Ed. Statistical Methods in Radio Wave Propagation, Elmsford, NY, Pergamon, 1960.

[6] M. D. Yacoub, D. B. da Costa, U. S. Dias, and G. Fraidenraich, "Joint Statistics for Two Correlated Weibull Variates," IEEE Antennas and Wireless Propagation Letters, vol. 4, pp. 129-132, 2005.

[7] J. Frolik, "On appropriate models for characterizing hyper-rayleigh fading," Wireless Communications, IEEE Transactions on, vol. 7, no. 12, pp. 5202-5207, 2008

[8] W. C. Jakes, Microwave Mobile Communications. IEEE Computer Society Press, 1994.

[9] L. Bakir and J. Frolik, "Diversity gains in two-ray fading channels," Wireless Communications, IEEE Transactions on, vol. 8, no. 2, pp. 968977, 2009.

[10] M. Yacoub, "The $\kappa-\mu$ distribution and the $\eta-\mu$ distribution," Antennas and Propagation Magazine, IEEE, vol. 49, no. 1, pp. 68-81, 2007.

[11] S. Cotton and W. Scanlon, "Higher-order statistics for $\kappa-\mu$ distribution," Electronics Letters, vol. 43, no. 22, 2007.

[12] I. S. Gradshteyn and I. M. Ryzhik, Table of Integrals, Series, and Products, Sixth Edition. Academic Press, 2000.

[13] J. Marcum, "A statistical theory of target detection by pulsed radar," Information Theory, IRE Transactions on, vol. 6, no. 2, pp. 59-267, 1960.

[14] M. Abramowitz and I. A. Stegun, Handbook of Mathematical Functions with Formulas, Graphs, and Mathematical Tables. New York: Dover, 1972.

[15] A. Papoulis, Probability, Random Variables, and Sthocastic Processes. McGraw-Hill, 1991. 